Results All procedures were completed laparoscopically. Follow-up at 6 months, 1, 2 and 3 years was $96 \%, 92 \%, 87 \%$ and $47 \%$ respectively. Mean (SD) \% excess weight loss at these time periods was 81.4 (25.6), 102.1 (27.3), 108.7 (38.2) and 97.5 (29.1) respectively. Mean (SD) weight loss at 2 years was $34.2(19.3) \mathrm{kg}$. $83 \%$ of patients demonstrated partial or complete resolution of their preoperative comorbidities including hypertension, diabetes, sleep apnoea and hyperlipidaemia. $17 \%$ developed new-onset reflux. $53 \%$ of patients required vitamin supplementation. Complications: Wound infection (1.8\%), food bolus obstruction requiring endoscopy (3.8\%) and silastic ring removal for food intolerance $(7.5 \%)$. There were no deaths.

Conclusion While the banded (silastic ring) SG is a safe procedure with good medium term excess weight loss and comorbidity resolution, around $11 \%$ of patients required endoscopic or surgical reintervention within 3 years.

Competing interests None declared.

\section{PM0-057 DIETARY INTERVENTION FOR INDIVIDUALS WITH SPINAL CORD INJURIES - A 2 YEAR REPORT ON THE SPINAL CLINIC FOR OBESE OUT-PATIENT PROJECT (SCOOP)}

doi:10.1136/gutjnl-2012-302514b.57

\begin{abstract}
${ }^{1,2} \mathrm{~S}$ S H Wong, ${ }^{*}{ }^{3} \mathrm{~A}$ Graham, ${ }^{3} \mathrm{~A}$ Jamous, ${ }^{1} \mathrm{~L}$ Spillman, ${ }^{2} \mathrm{G}$ Grimble, ${ }^{2} \mathrm{~A}$ Forbes. ${ }^{1}$ Department of Nutrition and Dietetics, National Spinal Injuries Centre, Aylesbury, UK; ${ }^{2}$ Centre for Gastroenterology and Clinical Nutrition, University College London, London, UK; ${ }^{3}$ National Spinal Injuries Centre, Stoke Mandeville Hospital, Aylesbury, UK
\end{abstract}

Introduction Obesity is common after spinal cord injuries (SCI) ${ }^{1}$ and it is associated with chronic nutrition related complications. We previously reported that a simple dietetic-intervention can help SCI patients to lose weight without compromising lean body mass. ${ }^{2}$

Methods The present study aimed to assess the effectiveness of a dietitian led weight management clinic. Thirty-one individuals (52.4 \pm 11.5 years; body mass index (BMI): $32.9 \mathrm{~kg} / \mathrm{m}^{2} ; 41 \%$ female) with SCI were referred for consultation to include nutritional advice and cognitive behaviour therapy in three consultations over 4 months. Outcomes measures included body composition from anthropometric measurements of BMI, mid upper-arm circumference (MUAC), triceps skinfold thickness (TSF) and mid-arm muscle circumference (MAMC), sitting blood pressure and dietary assessment of nutrient intake by 7-d food diary.

Results Of the thirty-one individuals, 15 (48.4\%) reported one or more co-morbidities. Mean weight loss was $4.5 \mathrm{~kg}$ (SD 6.2) at week 16. Analysis of the pre- to post-intervention difference (using paired t-test) showed a significant reduction in weight (104.2 vs $100.4 \mathrm{~kg}$, $\mathrm{p}=0.001$ ), BMI (34 vs $33.1 \mathrm{~kg} / \mathrm{m}^{2}, \mathrm{p}=0.004$ ), total energy intake (6.84 vs $5.62 \mathrm{MJ} / \mathrm{d}, \mathrm{p}=0.029)$, total fat $(\mathrm{p}=0.005)$, saturated fat $(p=0.001)$ and alcohol $(p=0.003)$. No significant changes were found in MUAC, TSF, MAMC and sitting blood pressure post intervention. We noted a significant reduction in MAMC at 1-year follow-up (30.2 vs $27.1 \mathrm{~cm}, \mathrm{p}<0.001)$. The 7-d food diary showed an average energy intake of $7.65 \mathrm{MJ} / \mathrm{d}$, which is $15 \%$ below the estimated requirement.

Conclusion The findings of this study suggest that current guidance overestimates nutritional requirements in the SCI population, and that simple dietetic-intervention could help overweight SCI patients to achieve weight loss, but that it may not be sufficient to stimulate/maintain muscle mass long term. Further research is warranted to test whether combining lifestyle and intensive physical therapy could reduce long-term muscle wasting.

Competing interests None declared.

\section{REFERENCES}

1. Gupta N, et al. Spinal Cord 2006:44:92-4.

2. Wong S, et al. Food Nutr Sci 2011;2:901-7.

\section{Parenteral nutrition}

\section{PM0-058 A SIMPLE AND AN EFFECTIVE STRATEGY FOR IMPROVING JUNIORS DOCTORS' KNOWLEDGE OF INTRAVENOUS FLUID THERAPY}

doi:10.1136/gutjnl-2012-302514b.58

${ }^{1}$ A P Swayamprakasam, ${ }^{*} \mathrm{P}$ Bijoor, ${ }^{1} \mathrm{U}$ Khalid, ${ }^{2} \mathrm{M}$ S Rana, ${ }^{1} \mathrm{R}$ Boulton, ${ }^{1} \mathrm{~A}$ Taylor. ${ }^{1}$ Department of General Surgery, Milton Keynes Hospital NHS Foundation Trust, Milton Keynes, UK; ${ }^{2}$ Department of Anaesthetics and Intensive Care, Milton Keynes Hospital NHS Foundation Trust, Milton Keynes, UK

Introduction Erroneous intravenous fluid therapy is a source of perioperative morbidity and mortality. ${ }^{1}$ In most surgical units, junior doctors are given the major responsibility for prescribing intravenous fluids, even though their knowledge base is inadequate. $^{2-4}$ Training junior doctors in intravenous fluid therapy is required to improve their knowledge and reduce the harm caused by erroneous intravenous fluid therapy. In this study we evaluated whether a single focused teaching session, based on the current national guidelines for perioperative fluid therapy, could improve junior doctors' knowledge of intravenous fluid therapy.

Methods Junior doctors attended a focused teaching session on a perioperative fluid therapy. They completed the questionnaire at baseline, immediately after and again $2-5$ weeks after the teaching session. The questionnaire was developed from the current national guidelines on perioperative intravenous fluid therapy; 2008 British Consensus Guidelines on Intravenous Fluid Therapy for Adult Surgical Patients.

Results The mean pre-education score was 4.3 (36\%). The mean post-education scores immediately after and an average of 22 days after the teaching session were; 10.3 (85\%) and 9.5 (79\%) respectively.

Conclusion A single focused teaching session is a simple and an effective way of improving junior doctors' knowledge of intravenous fluid therapy. We recommend that a teaching session on intravenous fluids be integrated into the teaching programme of all junior doctors.

Competing interests None declared.

\section{REFERENCES}

1. Callum KG, Gray AJG, Hoile RW, et al. Extremes of Age: The 1999 Report of the National Confidential Enquiry into Perioperative Deaths. London: National Confidentia Enquiry into Perioperative Deaths, 1999.

2. Lobo DN, Dube MG, Neal KR, et al. Problems with solutions: drowning in the brine of an inadequate knowledge base. Clin Nutr 2001:20:125-30.

3. Lobo DN, Dube MG, Neal KR, et al. Peri-operative fluid and electrolyte management: a survey of consultant surgeons in the UK. Ann R Coll Surg Engl 2002;84:156-60.

4. Walsh SR, Walsh CJ. Intravenous fluid-associated morbidity in postoperative patients. Ann R Coll Surg Eng/ 2005;87:126-30.

\section{PM0-059 PARENTERAL NUTRITION: AUDIT OF USAGE IN A DISTRICT GENERAL HOSPITAL WITHOUT A NUTRITION SUPPORT TEAM}

doi:10.1136/gutjnl-2012-302514b.59

${ }^{1}$ A Phillips, ${ }^{*}{ }^{2} \mathrm{~J}$ Brown, ${ }^{3} \mathrm{~K}$ Hinton, ${ }^{1} \mathrm{I}$ Gee. ${ }^{1}$ Department of Gastroenterology, Worcester Royal Hospital, Worcester, UK; ${ }^{2}$ Department of Nutrition and Dietetics, Worcester Royal Hospital, Worcester, UK; ${ }^{3}$ Department of Pharmacy, Worcester Royal Hospital, Worcester, UK

Introduction Parenteral nutrition (PN) is well known to have possibility of serious sequelae, in particular from catheter related sepsis (CRS). Multiple studies have demonstrated that a Nutrition Support Team (NST), in particular a Nutrition Support Nurse (NSN), can reduce inappropriate PN as well as reducing CRS rates (Kennedy et al 2005). The aim of this audit was to document PN usage in a district general hospital without a NST or NSN. 